\title{
Efficacy and Safety of Tadalafil Monotherapy for Lower Urinary Tract Symptoms Secondary to Benign Prostatic Hyperplasia: A Meta-Analysis
}

\author{
Yang Dong ${ }^{a}$ Lin Hao $^{b}$ Zhenduo Shi ${ }^{b}$ Gang Wang ${ }^{b}$ Zhiguo Zhang ${ }^{b}$ \\ Conghui Han ${ }^{a, b}$

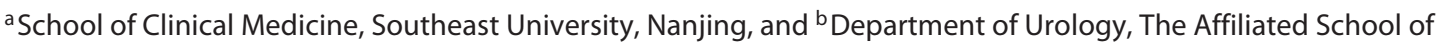 \\ Clinical Medicine, Xuzhou Medical College, Xuzhou, China
}

\section{Key Words}

Tadalafil · Cialis · Benign prostatic hyperplasia · Lower urinary tract symptoms $\cdot$ Meta-analysis

\begin{abstract}
Objective: To evaluate the efficacy and safety of tadalafil monotherapy for lower urinary tract symptoms secondary to benign prostatic hyperplasia (LUTS/BPH). Methods: A comprehensive search was done to identify randomized controlled trials comparing the efficacy and safety of tadalafil for LUTS/BPH with placebos. Meta-analytical techniques were applied to evaluate the differences in the study results. $\boldsymbol{R e}$ sults: Eight studies were identified and analyzed. Compared with placebo, tadalafil was associated with significant improvements in the International Prostate Symptom Score (IPSS) (mean difference $=-2.19, \mathrm{p}<0.00001$ ) and the International Index of Erectile Function (IIEF) score (mean difference $=+4.66, p<0.00001$ ), despite the concomitant presence of erectile dysfunction. Significant differences were also observed in the IPSS irritative and obstructive subscores, IPSS quality of life index and BPH impact index. After pooling four doses $(2.5,5,10$ and $20 \mathrm{mg})$, tadalafil failed to produce a significant outcome in maximal urinary flow rate $\left(\mathrm{Q}_{\max }\right)$ (mean difference $=+0.26 \mathrm{ml} / \mathrm{s}, p=0.14$ ), but $5 \mathrm{mg}$ of tadalafil
\end{abstract}

significantly improved $\mathrm{Q}_{\max }$ (mean difference $=+0.63 \mathrm{ml} / \mathrm{s}$, $p=0.04$ ). No significant difference was detected in the incidence of serious adverse events (risk ratio $=1.00, p=1.00$ ) after tadalafil treatment. Conclusions: Tadalafil showed good efficacy and safety for improving LUTS and erectile dysfunction in men with $\mathrm{BPH}$, and $5 \mathrm{mg}$ of tadalafil significantly improved $\mathrm{Q}_{\max }$.

Copyright $\odot 2013$ S. Karger AG, Basel

\section{Introduction}

Benign prostatic hyperplasia (BPH) is highly prevalent in elderly men and often results in lower urinary tract symptoms (LUTS), including urinary frequency, urgency, nocturia, intermittency, straining, incomplete emptying, and a weak urinary stream. LUTS secondary to BPH (LUTS/BPH) increases with age and negatively impacts patients' quality of life [1]. In addition to surgical interventions, the current standard treatments for LUTS/BPH consist of $\alpha 1$-adrenergic blockers, $5 \alpha$-reductase inhibi-

Y. Dong and L. Hao contributed equally to this work.

\section{KARGER}

E-Mail karger@karger.com

www.karger.com/uin
(C) 2013 S. Karger AG, Basel

0042-1138/13/0911-0010\$38.00/0
Conghui Han, $\mathrm{MD}$, $\mathrm{PhD}$

Department of Urology, The Affiliated School of Clinical Medicine Xuzhou Medical College

Xuzhou, 221009, Jiangsu Province (China)

E-Mail hanchdoctor@yahoo.cn 
tors and phytotherapies (used alone or in combination). Although efficacious, these therapies have the potential for side effects related to sexual dysfunction $[2,3]$.

Erectile dysfunction (ED) is a highly prevalent comorbidity in men with LUTS/BPH. The links between ED and LUTS/BPH have been identified in the Multinational Survey of the Aging Male [4] and in several epidemiologic studies [5], suggesting that the two diseases may share a common pathophysiology. The underlying mechanism for the relationship between LUTS and ED is still poorly understood, but common links such as RhoA/ Rho-kinase signaling, overactivity of the autonomic system, pelvic ischemia and the nitric oxide/cyclic guanosine monophosphate (NO/cGMP) pathway have been considered to be potential targets for phosphodiesterase type 5 inhibitors (PDE5-Is) [6-9].

PDE5-Is, including sildenafil (Viagra), vardenafil (Levitra) and tadalafil (Cialis), are widely approved for treating ED. Compared with the other PDE5-Is, tadalafil is unique in its longest half-life amounting to $17.5 \mathrm{~h}$, with drug efficacy potentially lasting up to $36 \mathrm{~h}$. No untoward concern of increased morbidity has been reported with its long half-life. Tadalafil is also unique in its higher selectivity for PDE11 compared to sildenafil or vardenafil; PDE11 is known to mainly present in the human prostate, testes and skeletal muscles, but its function is unclear. The special characteristics of tadalafil are, to a large part, attributed to its significantly different chemical structure compared with the other agents $[10,11]$. Recently, significant improvements in LUTS/BPH have been yielded by several clinical studies on PDE5-Is. Although the precise mechanism for ameliorating LUTS with PDE5-Is remains unclear, the proposed contributors include relaxing smooth muscle cells in the urogenital tract via the $\mathrm{NO} / \mathrm{cGMP} / \mathrm{PDE} 5$ pathway $[6,9]$.

At the present time, only two systematic reviews with meta-analyses using PDE5-Is in LUTS/BPH men are available online (August 2012) [7, 8]. Because of the dearth of randomized controlled trials (RCTs) examining each PDE5-I agent, different PDE5-Is (sildenafil, vardenafil and tadalafil) have all been included in previous meta-analyses. As reported, these agents had some different pharmacological profiles and side effects [10, 11], which might have affected the final conclusion. Regarding the use of tadalafil alone, significant improvements in LUTS/BPH or coexisting LUTS/BPH and ED have been recently reported by increasing numbers of RCTs. Therefore, conducting a meta-analysis of the current evidence was necessary to determine the relative efficacy and safety of monotherapy with tadalafil for LUTS/BPH.

\section{Materials and Methods}

\section{Search Strategy}

To identify relevant studies up to August 2012, we searched relevant electronic databases, including Medline, PubMed, Embase, the Cochrane Library database and the Web of Science. The websites of the European Association of Urology and the American Urological Association were searched for supplementary information. Ovid and Google Scholar were also used to find full-text articles. The search strategy included the following terms: tadalafil, IC351, IC-351, Cialis, lower urinary tract symptoms, LUTS, prostatic hyperplasia and $\mathrm{BPH}$. No methodological search filters were used and no date or language limits were applied. All citations of eligible articles and pertinent review articles were manually scanned to identify additional potentially relevant studies.

\section{Study Selection}

We performed this meta-analysis according to a prespecified protocol, which was guided by the PRISMA Statement and the Meta-analysis of Observational Studies in Epidemiology Consensus Statement. The following inclusion selection criteria were set: (1) the studies were RCTs; (2) the investigation was performed in humans; (3) the subject of the study was monotherapy with tadalafil for LUTS/BPH; (4) the control group received placebo alone; (5) the measured outcomes included at least one of the following: International Prostate Symptom Score (IPSS), International Index of Erectile Function (IIEF) score and maximal urinary flow rate $\left(\mathrm{Q}_{\max }\right)$. Studies were excluded if (1) they were not double-blinded, (2) the placebo was not identical in appearance or contained active drugs or (3) they were repetitive reports. We did not consider case reports, comments, meeting abstracts, letters to the editor, reviews or animal research. Only randomized double-blinded, placebo-controlled trials that evaluated the efficacy and safety of monotherapy with tadalafil for LUTS/BPH versus placebo were included. Two reviewers (Y.D. and L.H.) independently evaluated the literature that met the inclusion criteria and disagreements were resolved by consensus with a third reviewer who was blinded to the authors, journals and study source institutions.

\section{Data Extraction}

To assess the efficacy of tadalafil, the changes in IPSS, IIEF score and $\mathrm{Q}_{\max }$ from baseline to the endpoint after the tadalafil monotherapy were extracted as our primary outcomes. Our secondary outcomes were changes in the IPSS irritative and obstructive subscores, IPSS quality of life index (IPSS-QoL) and BPH impact index (BII). To assess the safety of tadalafil, all reported adverse events (AEs) in the selected trials were extracted, but only those reported in at least two papers were meta-analyzed.

The relevant data were extracted by two reviewers using a standard form to collect data. Another reviewer (C.H.) independently confirmed the accuracy of all the abstracted data. For studies with more than two interventions, each type of intervention with tadalafil versus placebo was extracted. When the necessary data were not shown in the article, we contacted the authors directly to obtain the original data, if possible.

\section{Statistical Analysis}

We evaluated the methodological quality of all the included trials with the Jadad score [12], and all statistical analyses were per- 


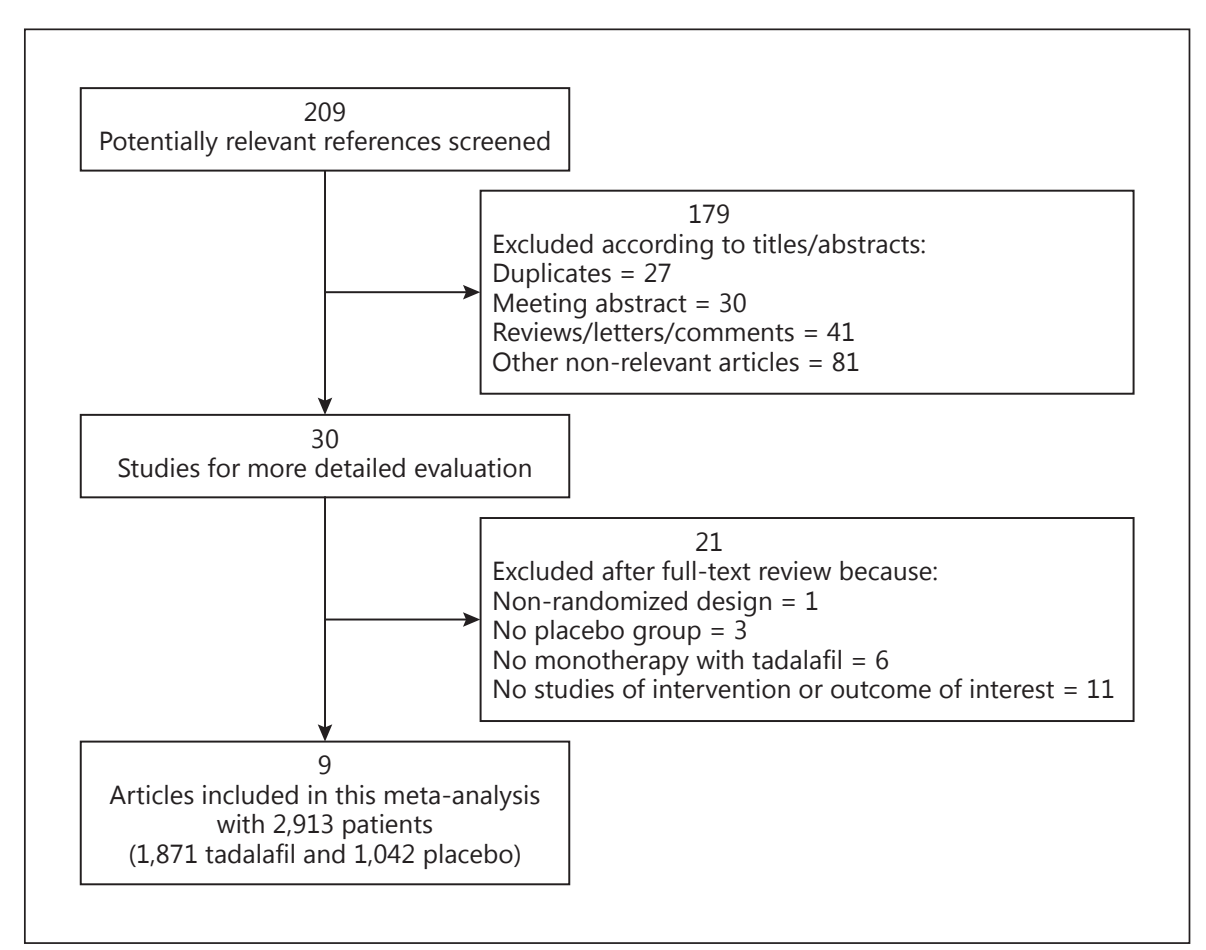

Fig. 1. Flow of included studies.

formed using Review Manager Version 5.0 (The Cochrane Collaboration, Oxford, UK). Publication bias was evaluated using a funnel plot, and the presence of heterogeneity among trials was assessed using $\chi^{2}$ and $\mathrm{I}^{2}$ statistics $\left(\mathrm{I}^{2}\right.$ value: $\leq 50 \%=$ low heterogeneity; $>50 \%$ to $<75 \%=$ moderate heterogeneity; $\geq 75 \%=$ high heterogeneity). In the case of a lack of heterogeneity, a fixed-effects model was used to estimate the pooled effect on the outcomes. However, when the heterogeneity was moderate or high, a random-effects model was used. The estimated effect measures were the mean difference for continuous data (e.g. the changes in the IPSS) and risk ratio for dichotomous data (e.g. AEs), and both were reported with $95 \%$ confidence intervals. Furthermore, for a more accurate analysis, a subgroup analysis was performed for some conditions.

\section{Results}

Flow of Included Studies and Study Characteristics

By using the search key terms listed earlier, 209 potentially relevant articles were identified. Finally, eight studies (from nine publications) [13-21] that enrolled 2,913 patients $(1,871$ tadalafil and 1,042 placebo) were included in the present meta-analysis. The process for the selection of relevant trials was performed as shown in figure 1 . The eight studies were published from 2007 to 2012. The characteristics and their quality are presented in table 1.

\section{Efficacy: Primary Outcomes}

Eight studies [13-21] evaluated the change in IPSS, and five studies $[13-15,19,20]$ evaluated the change in the IIEF score from baseline to the endpoint for tadalafil. According to the participants who reported having ED at baseline, the two analyses were divided into two subgroups: group $\mathrm{BPH}$ (patients with $\mathrm{BPH}$ ) and group $\mathrm{BPH} \& \mathrm{ED}$ (patients with comorbid $\mathrm{BPH}$ and $\mathrm{ED}$ ). In the subgroup analyses, tadalafil was associated with significant IPSS improvements in both group BPH $(2,267$ patients, -2.35 [ -2.83 to -1.88$], \mathrm{p}<0.00001)$ and group BPH\&ED (1,304 patients, -1.89 [-2.55 to -1.24$], \mathrm{p}<$ $0.00001)$. Tadalafil also remarkably improved the IIEF scores in group BPH $(1,226$ patients, +4.93 [3.74 to 6.13], $\mathrm{p}<0.00001)$ and group BPH\&ED (1,314 patients, +4.55 [3.79 to 5.32], $\mathrm{p}<0.00001)$. Combining the results, without heterogeneity $\left(\mathrm{I}^{2}=0 \%\right)$, tadalafil was superior to placebo for significantly ameliorating IPSS $(-2.19[-2.58$ to $-1.81], \mathrm{p}<0.00001$; fig. 2$)$ and the IIEF score $(+4.66$ [4.02 to 5.31$], \mathrm{p}<0.00001$; fig. 3 ).

The change in $\mathrm{Q}_{\max }$ from baseline was investigated in six studies $[13,16-19,21]$ including 2,188 patients with $\mathrm{BPH}$. Pooling all four doses $(2.5,5,10$ and $20 \mathrm{mg})$ showed that there was a non-significant difference in $\mathrm{Q}_{\max }$ for tadalafil versus placebo $(+0.26 \mathrm{ml} / \mathrm{s}[-0.09$ to 0.61$], \mathrm{p}=$ 0.14 ; fig. 4a). However, pooling only the data for tadalafil 
at a dose of $5 \mathrm{mg}$ (the dose most commonly tested for LUTS/BPH in four studies [17-19, 21] including 1,147 patients with $\mathrm{BPH}$ ) showed a significant improvement in $\mathrm{Q}_{\max }(+0.63 \mathrm{ml} / \mathrm{s}$ [0.03 to 1.22 ], $\mathrm{p}=0.04$; fig. $4 \mathrm{~b}$ ) as compared with placebo.

\section{Efficacy: Secondary Outcomes}

Improvements in the IPSS irritative and obstructive subscores were reported in six studies $[13,14,16,18-20]$ including 2,267 patients with $\mathrm{BPH}$. For the changes in the IPSS-QoL and BII, the data were derived from five [13, $14,16,18,20]$ and four $[13,14,16,20]$ studies that included 2,094 and 1,747 patients with $\mathrm{BPH}$, respectively. Heterogeneity was not observed $\left(\mathrm{I}^{2}=0 \%\right)$. The data were pooled for the calculations and computed for the combined analyses. Using the fixed-effect models, tadalafil significantly ameliorated IPSS irritative subscore $(-0.86$ [ -1.09 to -0.64$], \mathrm{p}<0.00001)$, IPSS obstructive subscore $(-1.47$ [ -1.78 to -1.16$], \mathrm{p}<0.00001)$, IPSS-QoL $(-0.35$ $[-0.45$ to -0.24$], \mathrm{p}<0.00001)$ and BII $(-0.49[-0.72$ to $-0.27], \mathrm{p}<0.0001)$ compared with placebo.

\section{Safety}

In studies comparing the safety of tadalafil versus placebo, 295 of 2,338 AEs (12.6\%) were reported in men treated with tadalafil compared with 56 of 1,157 AEs $(4.8 \%)$ in men treated with placebo. Table 2 lists the AEs that were reported in at least two trials. The data were pooled for the calculations. The most commonly reported AE was headache, followed by dyspepsia, back pain, nasopharyngitis and gastroesophageal reflux disease. The incidence of discontinuations due to AEs was 3.6\% (84) for tadalafil and $1.6 \%$ (18) for placebo. In addition, serious AEs were reported in $1.7 \%$ (32 patients) for tadalafil and $1.0 \%$ (11 patients) for placebo. Combining the results, the meta-analysis indicated that dyspepsia had the highest risk of occurrence after tadalafil administration versus placebo. In addition, there was no significant difference in the incidence of serious AEs between tadalafil and placebo using the fixed-effects model (table 2).

\section{Discussion}

As shown in our meta-analysis, tadalafil was superior to placebo for relieving LUTS/BPH and ED, as measured by IPSS (total, irritative and obstructive subscores and IPSS-QoL), BII and the IIEF score. The improvements appear to be clinically meaningful and are quite similar across the different doses of tadalafil. Although pooling

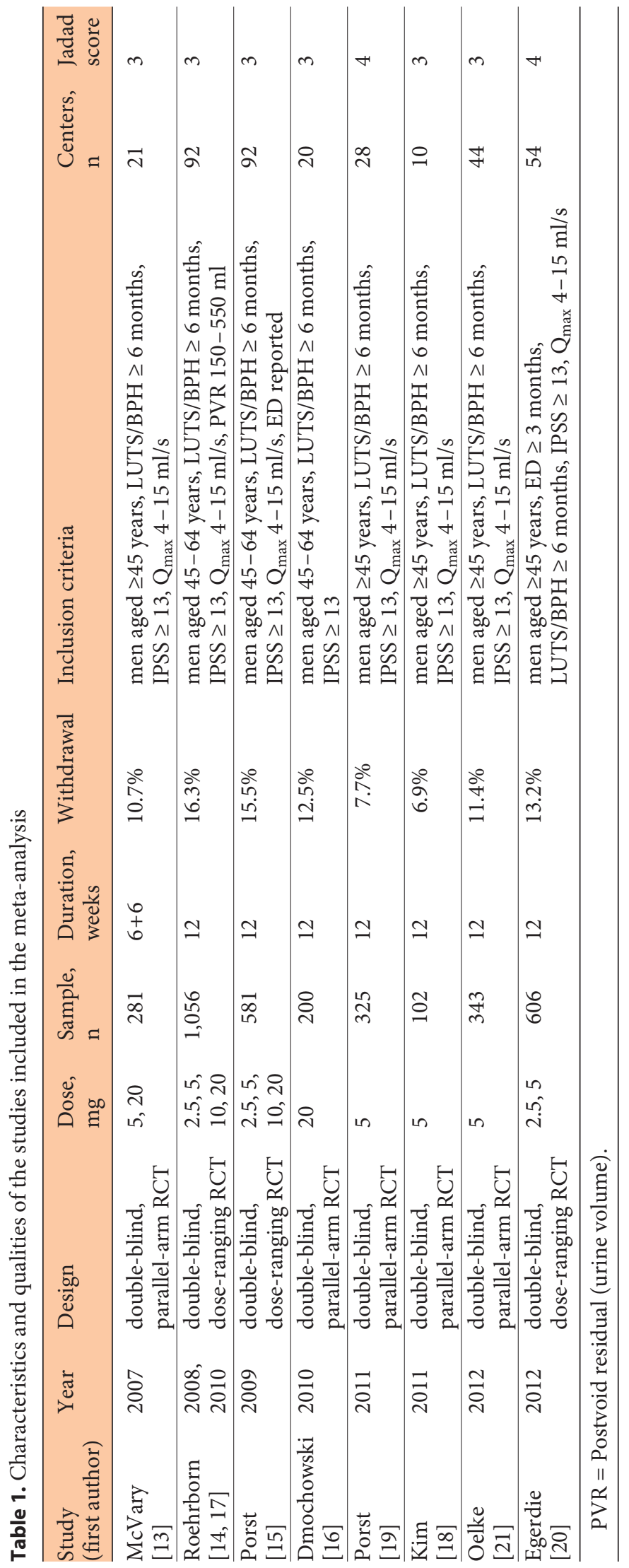




\begin{tabular}{|c|c|c|c|c|c|c|c|c|c|c|c|}
\hline \multirow{2}{*}{$\begin{array}{l}\text { Study } \\
\text { or subgroup }\end{array}$} & \multirow[t]{2}{*}{ Year } & \multicolumn{3}{|c|}{ Tadalafil } & \multicolumn{3}{|c|}{ Placebo } & \multirow{2}{*}{ Weight } & \multirow{2}{*}{$\begin{array}{l}\text { Mean difference } \\
\text { IV, fixed, } 95 \% \text { CI }\end{array}$} & \multirow{2}{*}{\multicolumn{2}{|c|}{$\begin{array}{l}\text { Mean difference } \\
\text { IV, fixed, } 95 \% \text { CI }\end{array}$}} \\
\hline & & mean & SD & total & mean & SD & total & & & & \\
\hline \multicolumn{12}{|l|}{ IPSS in BPH } \\
\hline McVary [13] & 2007 & -3.8 & 5.83 & 136 & -1.7 & 5.87 & 138 & $7.8 \%$ & $-2.10(-3.49,-0.71)$ & 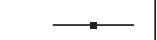 & \\
\hline Roehrborn [14] & 2008 & -4.87 & 7.13 & 212 & -2.27 & 7.1 & 210 & $8.1 \%$ & $-2.60(-3.96,-1.24)$ & $\longrightarrow$ & \\
\hline Roehrborn [14] & 2008 & -5.17 & 7.2 & 216 & -2.27 & 7.1 & 210 & $8.1 \%$ & $-2.90(-4.26,-1.54)$ & $\longrightarrow$ & \\
\hline Roehrborn [14] & 2008 & -3.88 & 7.21 & 208 & -2.27 & 7.1 & 210 & $7.9 \%$ & $-1.61(-2.98,-0.24)$ & $\longrightarrow$ & \\
\hline Roehrborn [14] & 2008 & -5.21 & 7.21 & 208 & -2.27 & 7.1 & 210 & $7.9 \%$ & $-2.94(-4.31,-1.57)$ & $\longrightarrow$ & \\
\hline Dmochowski [16] & 2010 & -9.2 & 6.9 & 82 & -5.1 & 7 & 89 & $3.4 \%$ & $-4.10(-6.18,-2.02)$ & & \\
\hline Porst [19] & 2011 & -5.8 & 4.24 & 50 & -4.2 & 4.24 & 50 & $5.4 \%$ & $-1.60(-3.26,0.06)$ & & \\
\hline Kim [18] & 2011 & -5.6 & 6 & 161 & -3.6 & 6 & 164 & $8.7 \%$ & $-2.00(-3.30,-0.70)$ & $\longrightarrow$ & \\
\hline Oelke [21] & 2012 & -6.3 & 6.5 & 171 & -4.2 & 6.6 & 172 & $7.7 \%$ & $-2.10(-3.49,-0.71)$ & & \\
\hline Subtotal $(95 \%$ CI) & & & & 1,444 & & & 1,453 & $65.0 \%$ & $-2.35(-2.83,-1.88)$ & & \\
\hline \multicolumn{12}{|c|}{ Heterogeneity: $\chi^{2}=6.60$, d.f. $=8(p=0.58), I^{2}=0 \%$} \\
\hline \multicolumn{12}{|c|}{ Test for overall effect: $Z=9.64(p<0.00001)$} \\
\hline \multicolumn{12}{|l|}{ IPSS in BPH\&ED } \\
\hline McVary [13] & 2007 & -4.4 & 6.26 & 80 & -1.8 & 6.02 & 74 & $4.0 \%$ & $-2.60(-4.54,-0.66)$ & & \\
\hline Porst [15] & 2009 & -4.7 & 8.39 & 110 & -2.1 & 8.5 & 113 & $3.0 \%$ & $-2.60(-4.82,-0.38)$ & & \\
\hline Porst [15] & 2009 & -4.7 & 8.54 & 114 & -2.1 & 8.5 & 113 & $3.0 \%$ & $-2.60(-4.82,-0.38)$ & & \\
\hline Porst [15] & 2009 & -3.6 & 8.31 & 108 & -2.1 & 8.5 & 113 & $3.0 \%$ & $-1.50(-3.72,0.72)$ & & \\
\hline Porst [15] & 2009 & -4.2 & 8.54 & 114 & -2.1 & 8.5 & 113 & $3.0 \%$ & $-2.10(-4.32,0.12)$ & & \\
\hline Egerdie [20] & 2012 & -6.1 & 5.74 & 206 & -3.8 & 6.96 & 194 & $9.5 \%$ & $-2.30(-3.55,-1.05)$ & 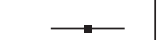 & \\
\hline Egerdie [20] & 2012 & -4.6 & 5.53 & 191 & -3.8 & 6.96 & 194 & $9.5 \%$ & $-0.80(-2.05,0.45)$ & & \\
\hline Subtotal $(95 \%$ CI) & & & & 923 & & & 914 & $35.0 \%$ & $-1.89(-2.55,-1.24)$ & & \\
\hline \multicolumn{12}{|c|}{$\begin{array}{l}\text { Heterogeneity: } \chi^{2}=4.77, \text { d.f. }=6(p=0.57), I^{2}=0 \% \\
\text { Test for overall effect: } Z=5.69(p<0.00001)\end{array}$} \\
\hline \multirow{2}{*}{\multicolumn{11}{|c|}{$\begin{array}{l}\text { Total }(95 \% \text { CI }) \\
\text { Heterogeneity: } x^{2}=12.61 \text {, d.f. }=15(p=0.63), I^{2}=0 \%\end{array}$}} & \\
\hline & & & & & & & & & & $\begin{array}{l}1 \\
-4\end{array}$ & $\frac{1}{2}$ \\
\hline \multicolumn{10}{|c|}{ Test for subgroup differences: $\chi^{2}=1.25$, d.f. $=1(p=0.26), I^{2}=19.7 \%$} & $\begin{array}{l}\text { Favors } \\
\text { tadalafil }\end{array}$ & $\begin{array}{l}\text { Favors } \\
\text { placebo }\end{array}$ \\
\hline
\end{tabular}

Fig. 2. Forest plot of change in IPSS from baseline to endpoint.

the doses at 2.5, 5, 10 and $20 \mathrm{mg}$ failed to result in a remarkable improvement in $\mathrm{Q}_{\max }$ in patients with $\mathrm{BPH}$, pooling only the data for $5 \mathrm{mg}$ of tadalafil significantly increased $\mathrm{Q}_{\max }$. This outcome provides evidence supporting the dose-dependent efficacy of tadalafil, and $5 \mathrm{mg}$ may be the optimum dose for treating LUTS/BPH.

Recently, a RCT [21] evaluating tadalafil and tamsulo$\sin$ (an a-blocker) for LUTS/BPH suggested that monotherapy with tadalafil or tamsulosin resulted in a significant and numerically similar improvement in IPSS versus placebo. However, only tadalafil significantly improved the IIEF score; tamsulosin did not. A similar outcome was also reported in a small pilot study of Korean men with LUTS/BPH [18]. In our meta-analysis, tadalafil significantly reduced the IPSS with a mean change of -4.61 . According to the American Urological Association guideline of a 3-point improvement in IPSS from baseline, the 4.61-point improvement is clinically relevant for symptomatic men and is perceived by patients [3]. This result also demonstrates that the IPSS reduction caused by tadalafil is comparable with that caused by a-blockers [22]. In addition, a significant improvement in the IIEF score was associated with tadalafil. Thus, tadalafil may be the optimum treatment for men suffering from LUTS comorbid with $\mathrm{BPH}$ and $\mathrm{ED}$.

Broderick et al. [23] compared the efficacy of tadalafil in $\mathrm{BPH}$ patients with and without ED and reported that the relief in LUTS caused by tadalafil was not influenced by ED. This finding revealed that the mechanism involved in LUTS/BPH in men with ED may not differ from that in men without ED. In our subgroup analyses, the mean change observed with tadalafil in IPSS in men with ED (mean change -1.89 ) or without ED (mean change -2.35 ) was similar to that of the overall population (mean 


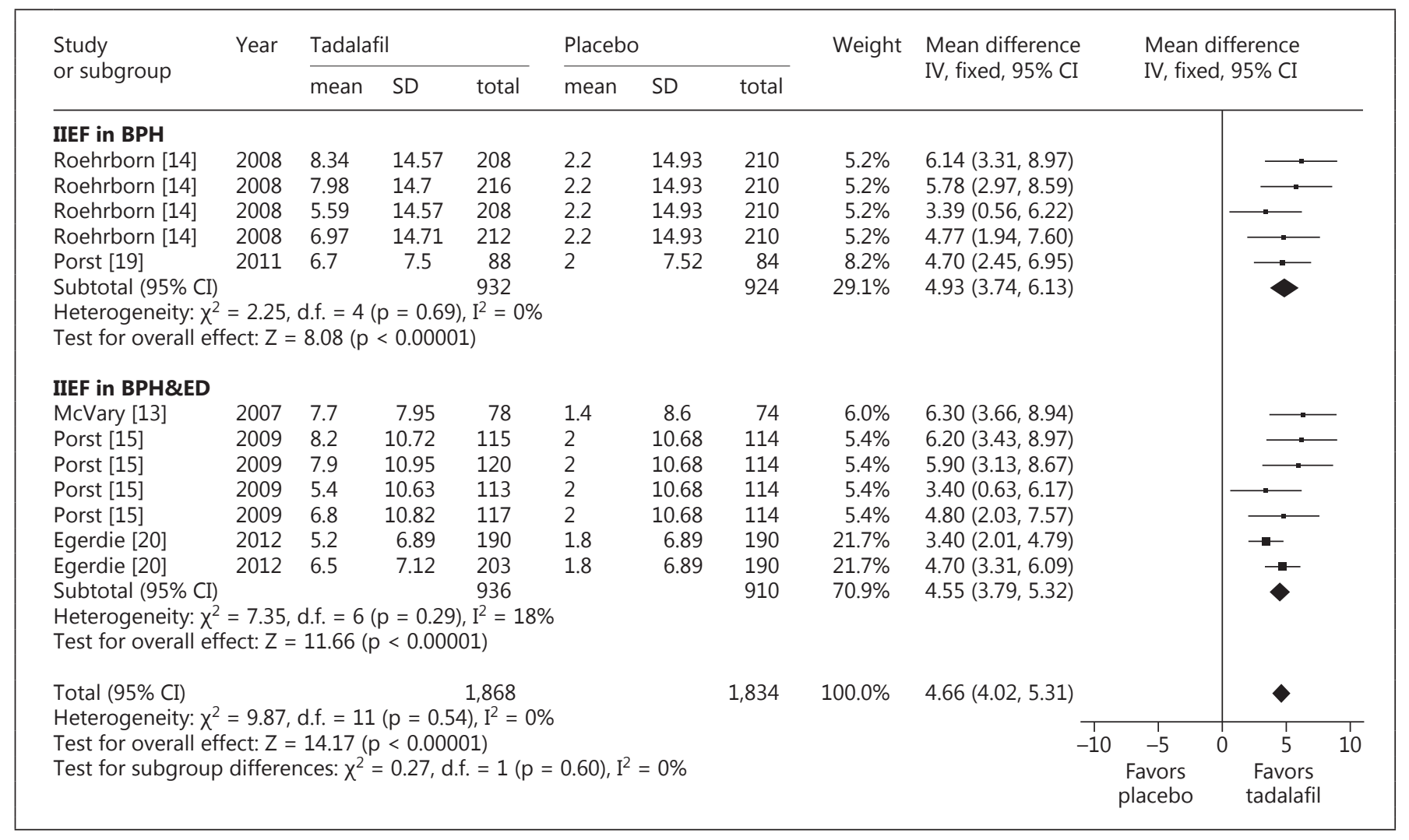

Fig. 3. Forest plot of change in IIEF score from baseline to endpoint.

change -2.19). Our result provides evidence supporting the above-mentioned conclusion and suggests that once daily tadalafil has a beneficial effect on IPSS, regardless of whether the patient has ED. Additionally, more significant improvements were found in the men without ED, indicating that the effect on LUTS was not predominantly mediated through its effect on ED.

The insignificant increase in $\mathrm{Q}_{\max }$ for all doses suggested that tadalafil can exert its clinical activity differently compared to the traditional BPH treatment with a-blockers. PDE5-Is act mainly to relieve the dynamic and static obstruction of the prostate with a direct relaxation of the bladder [8]. Several in vitro studies have reported smooth muscle relaxation in the human bladder neck and prostate in the presence of PDE5-Is [24, 25], and relaxation of the bladder neck and decreases in detrusor muscle overactivity have been detected in animal [26] and human studies [27]. Theoretically, the relaxation of the prostate and bladder neck after PDE5-I treatment could increase urinary flow; however, the concomitant relax- ation of the detrusor muscle counteracts this effect, thereby preventing the observation of a final improvement in $\mathrm{Q}_{\max }$ [27]. However, in a urodynamic study, tadalafil at a higher dose (20 mg once daily) had no adverse effect on the bladder outlet obstruction index in men with LUTS/ $\mathrm{BPH}$ [16]. Therefore, whether these theories and findings translate into the observed numeric $\mathrm{Q}_{\max }$ improvement observed with tadalafil remains unclear. Further basic studies are needed to determine the exact mechanisms involved in this field.

Different classes and doses of PDE5-Is may potentially affect the final results. Currently, no RCT has directly compared the efficacy of different classes of PDE5-Is, but the efficacy and safety of different tadalafil doses have been examined by several RCTs. In a dose-finding study, $5 \mathrm{mg}$ tadalafil was approved to provide a positive riskbenefit profile compared with other doses for 12 weeks [14]. In another long-term, dose-range study, the data suggested that over 12 months, $5 \mathrm{mg}$ was the most effective and safest dose [28]. In a 12-week study, Kang et al. 


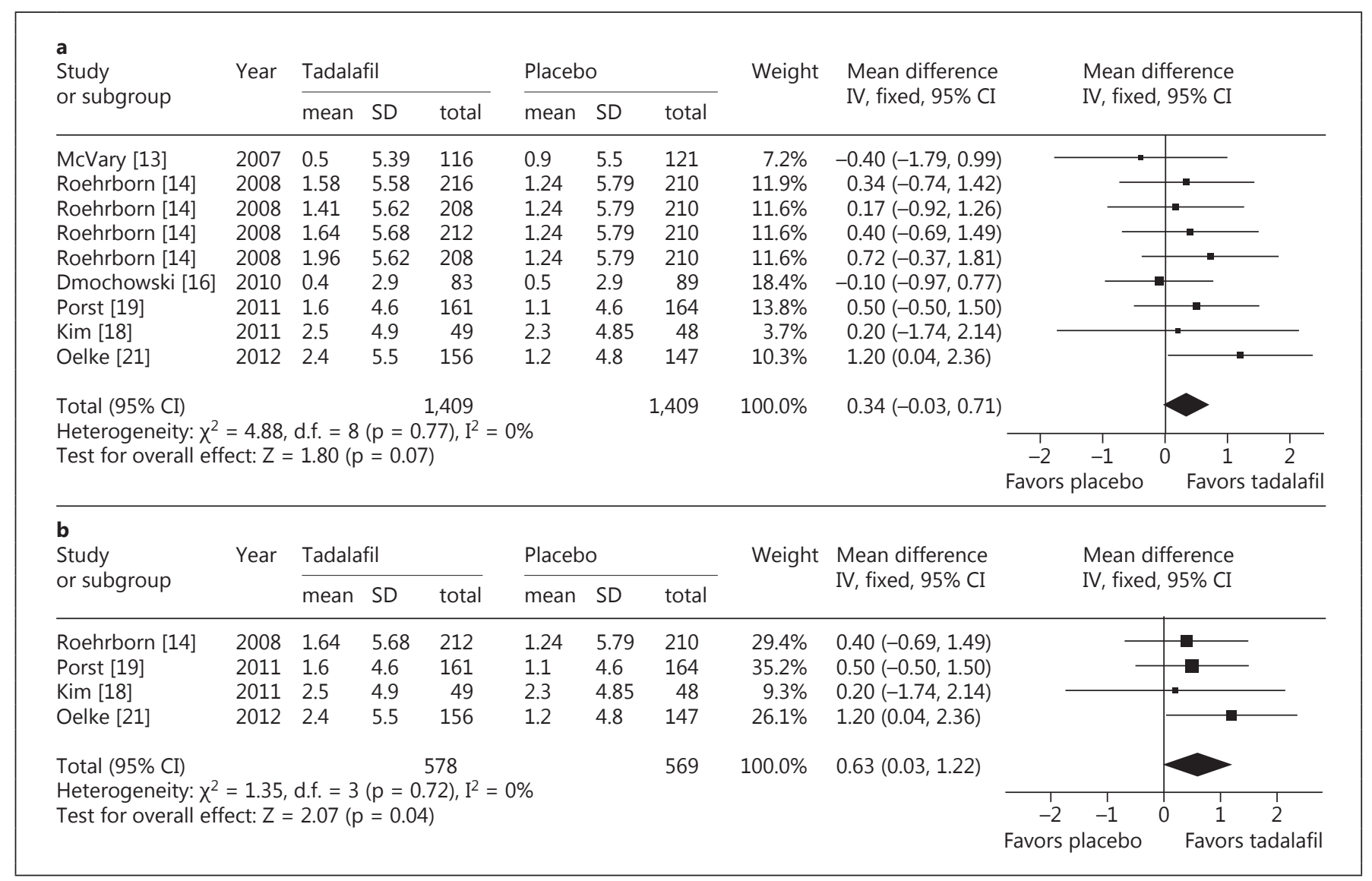

Fig. 4. a Forest plot of change in $\mathrm{Q}_{\max }$ from baseline to endpoint with 4 doses $(2.5,5,10$ and $20 \mathrm{mg})$ tadalafil in patients with $\mathrm{BPH}$. b Forest plot of change in $\mathrm{Q}_{\max }$ from baseline to endpoint with tadalafil dosed at $5 \mathrm{mg}$ in patients with BPH.

Table 2. Outcomes of the meta-analysis of AEs reported at least in two studies comparing the safety of monotherapy with tadalafil vs. placebo

\begin{tabular}{|c|c|c|c|c|c|c|}
\hline \multirow[t]{2}{*}{ Side effect } & \multirow{2}{*}{$\begin{array}{l}\text { Number } \\
\text { of studies }\end{array}$} & \multirow[t]{2}{*}{$\mathrm{RR}$} & \multirow[t]{2}{*}{$95 \% \mathrm{CI}$} & \multirow[t]{2}{*}{$\mathrm{p}$ value } & \multicolumn{2}{|c|}{ Incidence } \\
\hline & & & & & tadalafil & placebo \\
\hline Dyspepsia & 5 & 11.38 & $4.77-27.12$ & $<0.00001$ & $3.3 \%$ & $0 \%$ \\
\hline Back pain & 6 & 2.95 & $1.80-4.82$ & $<0.0001$ & $2.9 \%$ & $1.3 \%$ \\
\hline Gastroesophageal reflux & 2 & 7.40 & $1.98-27.67$ & 0.003 & $1.7 \%$ & $0 \%$ \\
\hline
\end{tabular}

$\mathrm{RR}=$ Risk ratio 95\% CI = 95\% confidence interval. 
[29] compared the improvements in LUTS by tadalafil at $20 \mathrm{mg}$ and $5 \mathrm{mg}$ once daily among $168 \mathrm{ED}$ patients and showed that the mean change in the IPSS was greater in the $5 \mathrm{mg}$ group $(-4.8 \pm 4.1)$ than in the $20 \mathrm{mg}$ group $(-2.3$ \pm 4.3 ). However, because of the limited numbers of patients receiving $5 \mathrm{mg}$ of tadalafil in these RCTs, we are unable to exclude the possibility that the lack of significant improvements in urinary flow rate may be attributed to the small sample size, which may be unable to detect small differences in the outcome. Unexpectedly, when we pooled these data, $5 \mathrm{mg}$ tadalafil showed a statistically significant increase in $\mathrm{Q}_{\max }(\mathrm{p}=0.04)$. Among these RCTs $[17-19,21]$, a numeric $Q_{\max }$ improvement was typically identified, but only Oelke et al. [21] reported a significant outcome in $\mathrm{Q}_{\max }(+2.4 \mathrm{ml} / \mathrm{s}, \mathrm{p}<0.05)$ in men with a lower baseline obstruction $\left(\mathrm{Q}_{\max }\right.$ at baseline: $\left.9.9 \mathrm{ml} / \mathrm{s}\right) \mathrm{com}$ pared with the other studies (range for tadalafil at $5 \mathrm{mg}=$ $9.8-11.7 \mathrm{ml} / \mathrm{s}$ ). The baseline urinary flow rate appears to determine the final result, and a lower baseline could allow more room for improvement, thereby increasing the probability of observing an improvement in $\mathrm{Q}_{\max }$.

Gacci et al. [8] reported that the degree of improvement in IPSS after PDE5-I treatment depended on the baseline characteristics of the patients, such as age, body mass index (BMI) and the baseline IPSS, indicating that young men with a low BMI and severe urinary symptoms (as measured by IPSS) are the best candidates for PDE5-I therapy. Aging and obesity appear to be associated with a testosterone decline, which can decrease the main target (PDE5) of PDE5-I in the bladder. Furthermore, defining a clear role for the baseline characteristics in the increase of $\mathrm{Q}_{\max }$ after PDE5-I treatment is strongly desirable.

Our meta-analysis showed a remarkable incidence of AEs with tadalafil. In contrast to the other PDE5-Is, flushing was not generally observed as an AE with the use of tadalafil. The inhibitory activity of tadalafil towards PDE11 has been speculated to be responsible for this side effect [25]. The results for the AEs are similar to those in previous long-term studies of once daily tadalafil treatment for LUTS/BPH [28] and ED [30]. The AEs were mild or moderate in severity and were transient. Serious AEs were reported in $1.23 \%$ of patients but did not show a significant difference between the tadalafil and placebo groups. Additionally, there was no clear pattern of differences in the incidence of AEs in men with or without ED, except for dyspepsia, which seemed to be more frequent in men without ED (risk ratio $=14.25$ ) than in those with $\mathrm{ED}$ (risk ratio $=6.41$ ) in the tadalafil group, as reported by Broderick et al. [23]. Overall, tadalafil is a safe treatment for patients with $\mathrm{BPH}$ or with comorbid $\mathrm{BPH}$ and $\mathrm{ED}$.
Several potential limitations should be considered in our meta-analysis. First, only four RCTs with a limited number of patients assessing the efficacy of $5 \mathrm{mg}$ tadalafil were included, and these insufficient data may thus affect the final conclusion, particularly with respect to the improvement in $\mathrm{Q}_{\max }$. Therefore, more RCTs testing tadalafil at this dose are needed, which will help draw a firm conclusion and establish an optimal dose for treatment. Second, we did not have access to the patient characteristics at baseline to determine whether the potential risk factors (age, BMI and other baseline parameters) could influence the results of the treatment. Third, the inclusion of a trial with a small sample size [18] or without a run-in period before the study [28] may affect the outcome assessment. Additionally, long-term efficacy and safety should be addressed by additional RCTs. Finally, the costeffectiveness of daily treatment with tadalafil should be evaluated, but no relevant studies are available. Therefore, further large-scale, high-quality RCTs are strongly desirable to ascertain the safety, efficacy and cost-effectiveness of once daily tadalafil in treating LUTS.

\section{Conclusions}

Tadalafil was shown to be significantly effective for improving LUTS/BPH, as measured by IPSS, the IIEF score, IPSS irritative and obstructive subscores, IPSSQoL and BII. Significant improvements in IPSS and the IIEF score were also observed in patients with comorbid $\mathrm{BPH}$ and $\mathrm{ED}$. When pooling all different doses of tadalafil, there was a numerical but insignificant improvement in $\mathrm{Q}_{\max }$, whereas pooling only the $5 \mathrm{mg}$ dose showed a significant improvement in $\mathrm{Q}_{\max }$. Additionally, AEs were transient and serious AEs were few, which demonstrated that tadalafil was well tolerated. Therefore, as a first-line treatment for ED, tadalafil can be considered as the optimum treatment for LUTS in patients with comorbid BPH and ED. Further high-quality studies are desirable to evaluate the optimal dose, relationship between the outcomes and characteristics of patients, long-term outcomes and overall cost-effectiveness of once daily tadalafil in treating LUTS/BPH.

\section{Acknowledgements}

We thank all of the authors of the primary studies included in the meta-analyses. 


\section{References}

$>1$ Wei JT, Calhoun E, Jacobsen SJ: Urologic diseases in America project: benign prostatic hyperplasia. J Urol 2005;173:1256-1261.

$>2$ Gacci M, Eardley I, Giuliano F, et al: Critical analysis of the relationship between sexual dysfunctions and lower urinary tract symptoms due to benign prostatic hyperplasia. Eur Urol 2011;60:809-825.

3 McVary KT, Roehrborn CG, Avins AL, et al: Management of benign prostatic hyperplasia (BPH). American Urological Association website: http://www.auanet.org/content/ guidelines-and-quality-care/clinical-guidelines.cfm?sub=bph (updated 2010).

4 Rosen R, Altwein J, Boyle P, et al: Lower urinary tract symptoms and male sexual dysfunction: the multinational survey of the aging male (MSAM-7). Eur Urol 2003;44:637649.

$\checkmark 5$ Rosen RC: Update on the relationship between sexual dysfunction and lower urinary tract symptoms/benign prostatic hyperplasia. Curr Opin Urol 2006;16:11-19.

6 Wang C: Phosphodiesterase-5 inhibitors and benign prostatic hyperplasia. Curr Opin Urol 2010;20:49-54.

$>7$ Liu L, Zheng S, Han P, et al: Phosphodiesterase-5 inhibitors for lower urinary tract symptoms secondary to benign prostatic hyperplasia: a systematic review and meta-analysis. Urology 2011;77:123-129.

-8 Gacci M, Corona G, Salvi M, et al: A systematic review and meta-analysis on the use of phosphodiesterase 5 inhibitors alone or in combination with alpha-blockers for lower urinary tract symptoms due to benign prostatic hyperplasia. Eur Urol 2012;61:994-1003.

$\checkmark 9$ Kedia GT, Uckert S, Jonas U, et al: The nitric oxide pathway in the human prostate: clinical implications in men with lower urinary tract symptoms. World J Urol 2008;26:603-609.

$>10$ Uckert S, Hedlund P, Andersson KE, et al: Update on phosphodiesterase (PDE) isoenzymes as pharmacologic targets in urology: present and future. Eur Urol 2006;50:11941207.

11 Carson CC 3rd: Phosphodiesterase type 5 inhibitors: state of the therapeutic class. Urol Clin North Am 2007;34:507-515, vi.

12 Jadad AR, Moore RA, Carroll D, et al: Assessing the quality of reports of randomized clinical trials: is blinding necessary? Control Clin Trials 1996;17:1-12.
13 McVary KT, Roehrborn CG, Kaminetsky JC, et al: Tadalafil relieves lower urinary tract symptoms secondary to benign prostatic hyperplasia. J Urol 2007;177:1401-1407.

14 Roehrborn CG, McVary KT, Elion-Mboussa A, et al: Tadalafil administered once daily for lower urinary tract symptoms secondary to benign prostatic hyperplasia: a dose finding study. J Urol 2008;180:1228-1234.

15 Porst H, McVary KT, Montorsi F, et al: Effects of once-daily tadalafil on erectile function in men with erectile dysfunction and signs and symptoms of benign prostatic hyperplasia. Eur Urol 2009;56:727-735.

16 Dmochowski R, Roehrborn C, Klise S, et al: Urodynamic effects of once daily tadalafil in men with lower urinary tract symptoms secondary to clinical benign prostatic hyperplasia: a randomized, placebo controlled 12week clinical trial. J Urol 2010;183:10921097.

17 Roehrborn CG, Kaminetsky JC, Auerbach $\mathrm{SM}$, et al: Changes in peak urinary flow and voiding efficiency in men with signs and symptoms of benign prostatic hyperplasia during once daily tadalafil treatment. BJU Int 2010;105:502-507.

18 Kim SC, Park JK, Kim SW, et al: Tadalafil administered once daily for treatment of lower urinary tract symptoms in Korean men with benign prostatic hyperplasia: results from a placebo-controlled pilot study using tamsulosin as an active control. Low Urin Tract Symptoms 2011;3:86-93.

19 Porst H, Kim ED, Casabe AR, et al: Efficacy and safety of tadalafil once daily in the treatment of men with lower urinary tract symp toms suggestive of benign prostatic hyperplasia: results of an international randomized, double-blind, placebo-controlled trial. Eur Urol 2011;60:1105-1113.

20 Egerdie RB, Auerbach S, Roehrborn CG, et al: Tadalafil 2.5 or $5 \mathrm{mg}$ administered once daily for 12 weeks in men with both erectile dysfunction and signs and symptoms of benign prostatic hyperplasia: results of a randomized, placebo-controlled, double-blind study. J Sex Med 2012;9:271-281.
21 Oelke M, Giuliano F, Mirone V, et al: Monotherapy with tadalafil or tamsulosin similarly improved lower urinary tract symptoms suggestive of benign prostatic hyperplasia in an international, randomised, parallel, placebocontrolled clinical trial. Eur Urol 2012;61: 917-925.

22 Wong P, Lawrentschuk N, Bolton DM: Phosphodiesterase 5 inhibitors in the management of benign prostatic hyperplasia and erectile dysfunction: the best of both worlds. Curr Opin Urol 2009;19:7-12.

23 Broderick GA, Brock GB, Roehrborn CG, et al: Effects of tadalafil on lower urinary tract symptoms secondary to benign prostatic hyperplasia in men with or without erectile dysfunction. Urology 2010;75:1452-1458.

24 Filippi S, Morelli A, Sandner P, et al: Characterization and functional role of androgendependent PDE5 activity in the bladder. Endocrinology 2007;148:1019-1029.

25 Morelli A, Sarchielli E, Comeglio P, et al: Phosphodiesterase type 5 expression in human and rat lower urinary tract tissues and the effect of tadalafil on prostate gland oxygenation in spontaneously hypertensive rats. J Sex Med 2011;8:2746-2760.

26 Beamon CR, Mazar C, Salkini MW, et al: The effect of sildenafil citrate on bladder outlet obstruction: a mouse model. BJU Int 2009;104: 252-256.

27 Gacci M, Del Popolo G, Macchiarella A, et al: Vardenafil improves urodynamic parameters in men with spinal cord injury: results from a single dose, pilot study. J Urol 2007;178: 2040-2043; discussion 2044.

28 Donatucci CF, Brock GB, Goldfischer ER, et al: Tadalafil administered once daily for lower urinary tract symptoms secondary to benign prostatic hyperplasia: a 1-year, open-label extension study. BJU Int 2011;107:1110-1116.

-29 Kang DH, Lee JY, Chung JH, et al: Comparison of efficacy for erectile function and lower urinary tract symptoms of tadalafil $20 \mathrm{mg}$ ondemand and $5 \mathrm{mg}$ once daily in patients with erectile dysfunction. Int J Clin Pract 2012;66: 813-820.

30 Porst H, Rajfer J, Casabe A, et al: Long-term safety and efficacy of tadalafil $5 \mathrm{mg}$ dosed once daily in men with erectile dysfunction. J Sex Med 2008;5:2160-2169. 